\title{
Human induced pluripotent stem cell-derived mesenchymal stem cells alleviate atherosclerosis by modulating inflammatory responses
}

\author{
HUI SHI ${ }^{1,2 *}$, MEILING LIANG ${ }^{3 *}$, WEIYAN $\mathrm{CHEN}^{4}$, XIUTING SUN $^{1}$, XIANG WANG $^{5}$, \\ CHENGHSUN LI $^{1}$, YIYING YANG ${ }^{1}$, ZHISHENG YANG ${ }^{1}$ and WUTAO ZENG ${ }^{1,2}$
}

\begin{abstract}
${ }^{1}$ Department of Cardiology, Heart Center, The First Affiliated Hospital, Sun Yat-sen University, Guangzhou, Guangdong 510080; ${ }^{2}$ Key Laboratory on Assisted Circulation, Ministry of Health, Guangzhou; ${ }^{3}$ Department of Cardiology, Sun Yat-sen Cardiovascular Hospital of Shenzhen, Shenzhen; ${ }^{4}$ Department of Intensive Care Unit, The Second Affiliated Hospital of Guangzhou Medical University, Guangzhou, Guangdong 510080;

${ }^{5}$ Department of Cardiology, Laiwu People's Hospital, Laiwu, Shandong 271100, P.R. China
\end{abstract}

Received October 29, 2016; Accepted June 13, 2017

DOI: $10.3892 / \mathrm{mmr} .2017 .8075$

\begin{abstract}
The transplantation of mesenchymal stem cells (MSCs) has been a reported method for alleviating atherosclerosis (AS). Because the availability of bone marrow-derived MSCs (BM-MSCs) is limited, the authors used this study to explore the use of a new type of MSC, human induced pluripotent stem cell-derived MSCs (iPSC-MSCs), to evaluate whether these cells could alleviate AS. iPSC-MSCs were intravenously administered to ApoE knock out mice fed on a high-fat diet (HFD) for 12 weeks. It was reported that systematically administering iPSC-MSCs clearly reduced the size of plaques. In addition, the numbers of macrophages and lipids in plaques were lower in the HFD + iPSC-MSCs group than in the HFD group. Furthermore, iPSC-MSCs attenuated AS-associated inflammation by decreasing the levels of inflammatory cytokines, such as tumor necrosis factor- $\alpha$ and interleukin-6, in serum. In addition, the expression of Notch1 was higher in the HFD group, and injecting iPSC-MSCs reversed this effect. In conclusion, the current study provides the first evidence indicating that iPSC-MSCs may be a new optional MSC-based strategy for treating AS.
\end{abstract}

Correspondence to: Professor Wutao Zeng, Department of Cardiology, Heart Center, The First Affiliated Hospital, Sun Yat-sen University, 58 Zhongshan 2nd Road, Yuexiu, Guangzhou, Guangdong 510080, P.R. China

E-mail: zengwutao_sysumed@163.com

*Contributed equally

Key words: induced pluripotent stem cell, mesenchymal stem cells, atherosclerosis, inflammatory responses

\section{Introduction}

Atherosclerosis (AS) is a leading cause of illness around the world that is widely considered to represent a chronic inflammatory disease (1) and has been associated with many cardiovascular events (2). It was previously reported that macrophages accumulate in the intima, leading to the formation of foam cells, which are the major hallmark of early-stage atherosclerotic lesions (3). Foam cells secrete many inflammatory cytokines, such as tumor necrosis factor (TNF)- $\alpha$ and interleukin (IL)-6, that have emerged as key players in the pathogenesis of AS (4). These inflammatory cytokines induce a cascade that leads to the further aggravation of AS (5).

Mesenchymal stem cells (MSCs) are multipotent cells that possess immunomodulatory potential (6). They are widely used in preliminary clinical trials and animal studies, including animal models of AS (7-9). It was previously reported that bone marrow-derived mesenchymal stem cells (BM-MSCs) attenuated AS formation and inflammatory responses $(7,10)$. However, despite the benefits provided by BM-MSCs, they are associated with some shortcomings, such as cellular senescence, limited proliferative capacity and rapid loss of differentiation potential $(11,12)$. One previous breakthrough in the field is the generation of induced pluripotent stem cell (iPSCs) $(13,14)$, which provides a new source of MSCs and has therefore attracted worldwide attention. When systematically administered into limb-ischemia mice, iPSC-MSCs improved the limb salvage rate (15). Moreover, iPSC-MSCs also alleviated inflammatory reactions in allergic airway inflammation mice by reducing inflammatory cell infiltration and the levels of inflammatory cytokines (16). Additionally, iPSC-MSCs have longer survival times, stronger proliferative capacity and more stable cell properties than BM-MSCs (17), indicating that iPSC-MSCs may be better candidates for future therapies. The therapeutic efficacy of iPSC-MSCs in AS has not been previously demonstrated. In the present study, the authors used a mouse model of AS ApoE knockout (KO) mice. Following 
this, the effects of infusing iPSC-MSCs on plaque pathology and inflammatory responses were then evaluated in these mice.

\section{Materials and methods}

Materials and regents. ApoE-/- mice were purchased from Beijing Vital River Laboratories Animal Technology Co., Ltd. (Beijing, China). The high-fat diet (sugar 20\%, lard 15\%, cholesterol $1.2 \%$, sodium cholate $0.2 \%$, casein $10 \%$, corn $25 \%$, bran $13 \%$, soybean $15 \%$, salt $0.6 \%$ ) was provided by Guangdong Medical Laboratory Animal Center (Guangzhou, China). The iPSC-MSCs were generously provided by Dr Qizhou Lian of Hong Kong University (Hong Kong). FBS and Dulbecco's modified Eagle's medium (DMEM) were purchased from Gibco; Thermo Fisher Scientific, Inc. (Waltham, MA, USA). Oil red O was obtained from Sigma-Aldrich; Merck KGaA (Darmstadt, Germany). The anti-GAPDH rabbit monoclonal antibody (mAb; cat. no. 2118) and anti-rabbit IgG, horseradish peroxidase (HRP)-linked antibody (cat. no. 7074) were supplied by Cell Signaling Technology, Inc. (Danvers, MA, USA). Anti-Notch1 rabbit mAb (cat. no. ab52627) and anti-CD68 mouse mAb (cat. no. ab955) were purchased from Abcam (Cambridge, UK). Mouse IL-6 ELISA kit (cat. no. CSB-E04639m) and mouse TNF- $\alpha$ ELISA kit (cat. no. CSB-E04741m) were provided by CUSABIO (Wuhan, China).

Animals. A total of 32 male ApoE-/- mice (C57BL/6 background, 7 weeks old) were housed under a $12 \mathrm{~h}$ light-dark cycle in the Sun Yat-Sen University Laboratory Animal Center (Shenzhen, China). Following acclimatization for one week, they were randomly divided into the following four groups: The RD group comprised the regular diet group, which did not receive any treatment $(n=8)$; the HFD group was fed a high-fat diet $(n=8)$; the HFD + iPSC-MSCs group was fed a high-fat diet and received $400 \mu 1$ PBS containing $2 \times 10^{6}$ iPSC-MSCs $(\mathrm{n}=8)$; and the HF + PBS group was fed a high fat diet and received $400 \mu 1 \mathrm{PBS}(\mathrm{n}=8)$. Stem cells and PBS were injected via the tail vein every 7-10 days. After 12 weeks, the animals were anesthetized using $1.5 \%$ pentobarbital. Blood was obtained from the right ventricle and centrifuged at 1,006 x g for $10 \mathrm{~min}$ to isolate serum. Aortas and liver tissue were carefully harvested from the mice. Aortic arches and liver tissue were used for histology, and the remaining tissue was frozen in liquid nitrogen and stored at $-80^{\circ} \mathrm{C}$ in a freezer until used. All animal care and treatment procedures were approved by the Local Animal Care Committee and conformed to Institutional Guidelines and Animal Ordinances (Zhongshan School of Medicine, Sun Yat-sen University, Shenzhen, China).

Cell culture. Human iPSC-MSCs derived from iPSC lines have been described previously $(15,18)$. The iPSC-MSCs resemble BM-MSCs in morphology and have similar surface antigen expression, such as CD44, CD73, CD105 and CD166 but lack CD45, CD34 and CD133. In addition, iPSC-MSCs also have the capacity to differentiate into osteoblasts, adipocytes and chondroblasts. iPSC-MSCs are cultured in DMEM supplemented with $10 \% \mathrm{FBS}$ at $37^{\circ} \mathrm{C}$ in an atmosphere containing $5 \% \mathrm{CO}_{2}$ and $95 \%$ air. Cells in passages 10-15 were injected into the mice via the tail vein.
En face preparation. Following isolation, the aortas were cut longitudinally and fixed onto dishes. The aortas were stained with Oil-Red $\mathrm{O}$ for 30-40 min and then rinsed with $70 \%$ ethanol. Images of en face aortas were acquired using a camera. The sizes of the stained areas were calculated using Image-Plus Pro 5.0 (Media Cybernetics, Inc., Rockville, MD, USA).

Histological stains. The aortic arches were used to determine the area affected by atherosclerotic lesions. After the arches were dissected from the aorta, they were fixed in 4\% paraformaldehyde overnight and embedded in paraffin. The embedded aortas were sliced into $5 \mu \mathrm{m}$-thick sections. The sections were then stained using hematoxylin and eosin (Wuhan Boster Biological Technology, Ltd., Wuhan, China). The average lesion size was determined using measurements obtained from 10 serial sections. Liver sections were stained with Oil Red O. All images were obtained with the inverted optical microscope (BX51; Olympus Corporation, Tokyo, Japan), The sizes of the stained areas were calculated using Image-Plus Pro5.0 (Media Cybernetics, Inc.).

Immunohistochemical study. The paraffin-embedded sections were deparaffinized and rehydrated. They were then processed for antigen retrieval in a microwave oven. The sections were then rinsed in PBS containing 0.1\% Tween-20 (PBS-T) and blocked in $3 \%$ peroxide-methanol at room temperature to inhibit endogenous peroxidase activity. The sections were blocked in 3\% bovine serum albumin (Invitrogen; Thermo Fisher Scientific, Inc.), for $1 \mathrm{~h}$ at room temperature and then incubated with primary antibodies for CD68 (dilution, 1:200) overnight at $4^{\circ} \mathrm{C}$. Following this, the sections were then incubated with HRP-conjugated anti-rabbit secondary antibody (dilution, 1:100) for $50 \mathrm{~min}$ at room temperature, color was developed using 3,3-diaminobenzidine (Wuhan Boster Biological Technology, Ltd.). After the sections were counterstained with hematoxylin (Wuhan Boster Biological Technology, Ltd.), the slides were sequentially washed through an alcohol dehydration series and mounted using neutral gum. The slides were examined using the inverted optical microscope (BX51; Olympus Corporation), The sizes of the stained areas were calculated using Image-Plus Pro5.0 (Media Cybernetics, Inc.).

Measurement of serum lipid levels. Serum samples were obtained to measure serum cholesterol levels, including the levels of total cholesterol, low-density lipoprotein (LDL) and high-density lipoprotein (HDL), using an autoanalyzer (Cobas Integra 400, Roche Diagnostics, Indianapolis, IN, USA).

ELISA assay. Serum was collected as described above. IL-6 and TNF- $\alpha$ levels were measured according to the instructions that accompanied the appropriate ELISA kits. Every result was obtained but combining the results from 8 mice in each group.

Western blot analysis. The aortas were used to examine the protein expression. Total proteins were extracted from aortas using radioimmunoprecipitation assay buffer (Cell Signaling Technology, Inc.). Following centrifugation at $12,000 \mathrm{x} \mathrm{g}$ at $4^{\circ} \mathrm{C}$ for $10 \mathrm{~min}$, the supernatant was collected. The bicinchoninic 

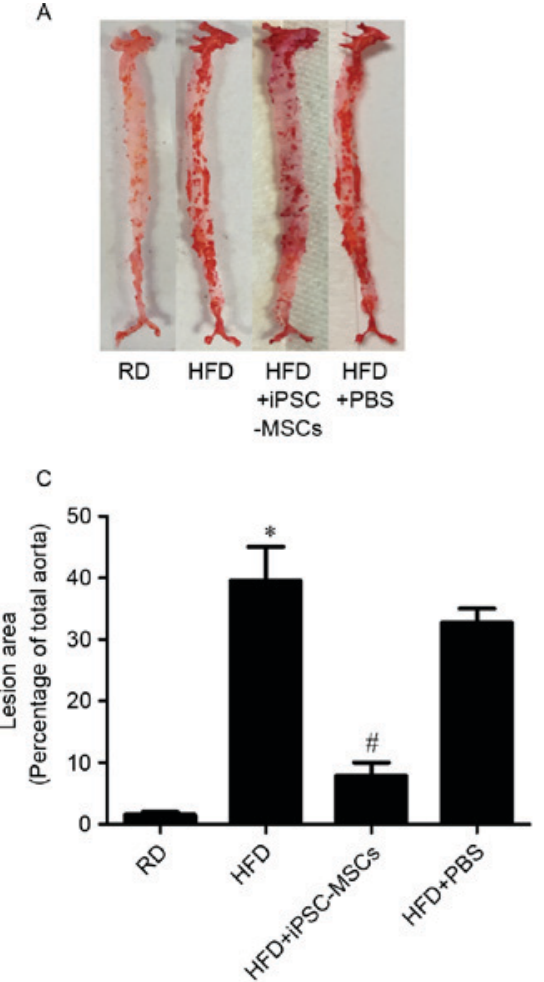

B

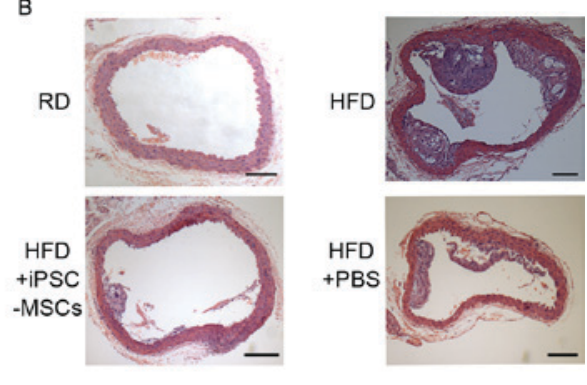

D

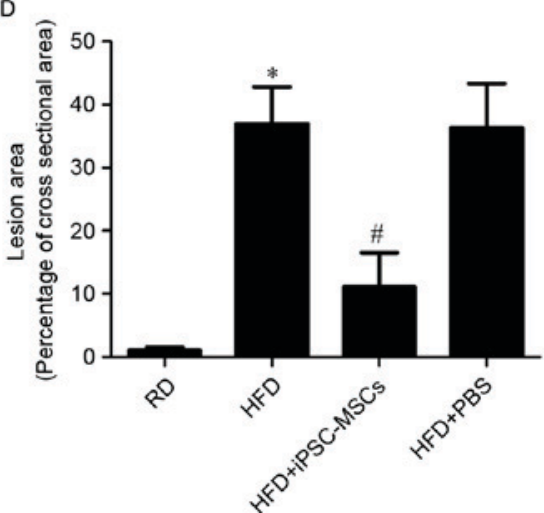

Figure 1. Infusion of iPSC-MSCs alleviated the atherosclerotic plaque formation (A) Morphology of en face surface lesions. (B) Representative photomicrographs of hematoxylin and eosin staining of cross sectional areas of aortic roots. (C) Quantification of surface lesion areas. (D) Quantification of cross sectional plaque areas. Data are presented as mean \pm standard error of the mean. ${ }^{*} \mathrm{P}<0.01$ vs. RD group; ${ }^{*} \mathrm{P}<0.05$ vs. HFD group. Scale bar, $500 \mu \mathrm{m}$. iPSC-MSCs, induced pluripotent stem cell-derived mesenchymal stem cells; RD, regular diet group; HFD, high-fat diet group; PBS, phosphate-buffered saline.

acid protein assay kit (Pierce; Thermo Fisher Scientific, Inc.) was used to quantify the protein concentrations. Protein $(20 \mu \mathrm{g})$ from each group were fractionated on $8 \%$ SDS-PAGE gels (Amresco,LLC, Solon, OH, USA). The proteins were transferred into polyvinylidene difluoride membrane (Roche Diagnostics). Then the membranes were blocked with $5 \%$ fat-free milk at room temperature for $1 \mathrm{~h}$ in TBS with $0.1 \%$ Tween-20, and incubated with anti-notch1 (dilution, 1:1,000), in TBS containing $0.1 \%$ Tween-20 (TBS-T) with $5 \%$ BSA at $4^{\circ} \mathrm{C}$ overnight. Membranes were washed with TBS-T for 5 min three times, and incubated with HRP-conjugated secondary antibody (dilution, 1:2,000) for $60 \mathrm{~min}$ at room temperature. After washing, protein bands on the membrane were visualized by enhanced chemiluminescence (EMD Millipore, Billerica, MA, USA). The quantitative analysis of the blot intensities was performed using Quantity One 4.62 software (Bio-Rad Laboratories, Inc., Hercules, CA, USA).

Statistical analysis. All data were analyzed using SPSS software (version, 16.0; SPSS Inc., Chicago, IL, USA). The data are presented as the mean \pm standard error of the mean. Differences among the four groups were determined using one way analysis of variance followed by the SNK-q test. $\mathrm{P}<0.05$ was considered to indicate a statistically significant difference.

\section{Results}

iPSC-MSCs reduced atherosclerotic plaque formation. To determine whether iPSC-MSCs affect AS, the authors used en face and cross sections to measure the sizes of atherosclerotic plaques in the aortas of ApoE KO mice. Using Oil Red O staining (Fig. 1A), they reported that a significantly larger area was covered by plaques in the ApoE KO mice fed on a HFD than in those in the RD group (Fig. 1C; $\mathrm{P}<0.01$ ). Administering iPSC-MSCs reversed this effect of a HFD by decreasing the plaque area to $7 \%$ (Fig. 1C; $\mathrm{P}<0.05$ ). However, the sizes of the plaques were not different between the HF and PBS groups (Fig. 1C; $\mathrm{P}>0.05$ ). Consistent with the en face results, in cross sections (Fig. 1B), the authors indicated that smaller plaque sizes were observed in the iPSC-MSCs group than in the HFD group (Fig. 1D; $\mathrm{P}<0.05$ ). No significant differences were detected between the HFD and PBS groups (Fig. 1D; P>0.05). These results indicated that infusions of iPSC-MSCs inhibited the formation of atherosclerotic plaques. In addition, GFP-labeled iPSC-MSCs were injected into ApoE $\mathrm{KO}$ mice, and a previous study demonstrated that iPSC-MSCs are capable of migrating to impaired sites to exert their protective functions (19). Unfortunately, the authors failed to detect GFP-labeled iPSC-MSCs in inflamed tissues, indicating that the injected iPSC-MSCs may not alleviate AS through a direct effect.

iPSC-MSCs decreased the number of macrophages and lipids in plaques. The authors next used Oil Red $\mathrm{O}$ to stain frozen sections to determine the lipid content in plaques (Fig. 2A). Administering iPSC-MSCs resulted in significantly lower lipid levels in plaques than were observed in the HFD-only group (Fig. 2B; $\mathrm{P}<0.05$ ). Immunohistochemical analysis indicated that the iPSC-MSC-treated mice displayed a profound change in macrophage infiltration in plaques (Fig. 2A). There were significantly fewer macrophages (CD68-positive cells) 

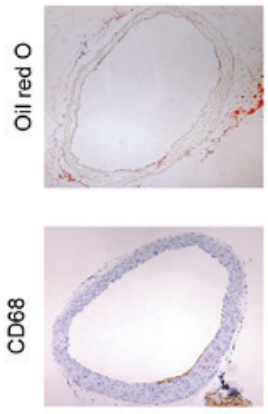

RD
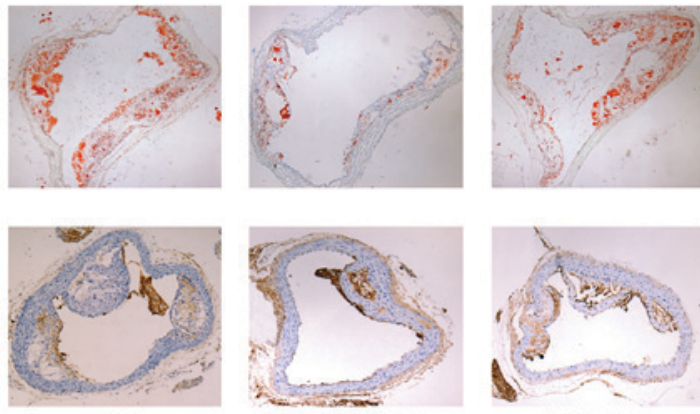

HFD

B

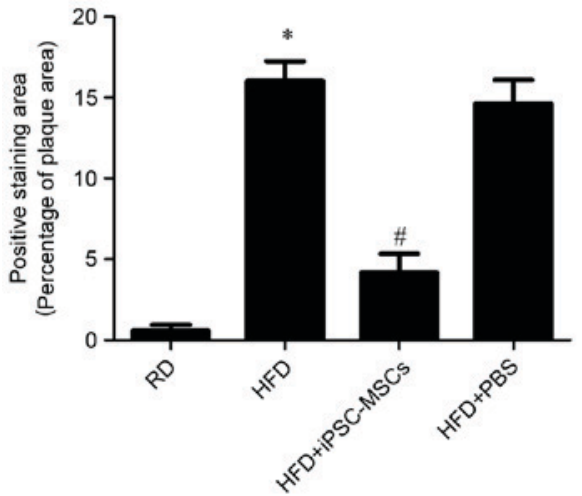

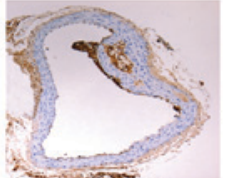

HFD+iPSC-MSCs

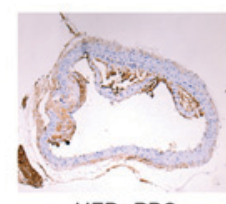

HFD+PBS

C

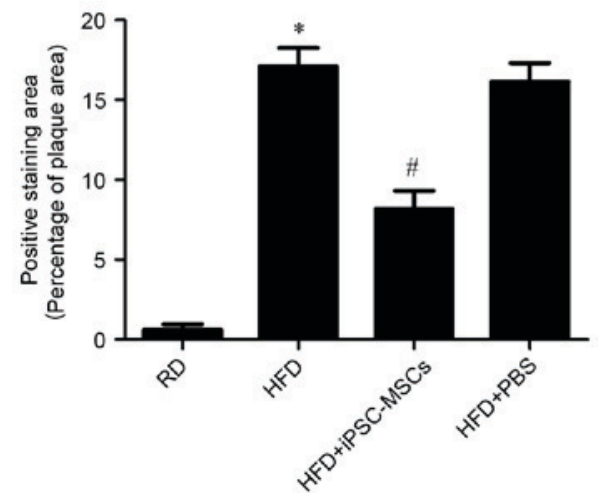

Figure 2. iPSC-MSCs' effect on the plaque components. (A) Representative immunohistochemical staining of lipids (Oil red O), macrophages (CD68) of the aortic root plaques. Magnification, x100. (B) Quantitative analysis of lipids. (C) Quantitative analysis of macrophages. Data are presented as mean \pm standard error of the mean. "P<0.01 vs. RD group; ${ }^{\#} \mathrm{P}<0.01$ vs. HFD group. Scale bar, $500 \mu \mathrm{m}$. iPSC-MSCs, induced pluripotent stem cell-derived mesenchymal stem cells; RD, regular diet group; HFD, high-fat diet group; PBS, phosphate-buffered saline.

in plaques in the mice infused with iPSC-MSCs than in the HFD-only group (Fig. 2C; $\mathrm{P}<0.05$ ). Additionally, there was no difference in the lipid content or the number of CD68-positive cells between the HFD and PBS groups (Fig. 2B and C; $\mathrm{P}>0.05)$. It was therefore inferred that iPSC-MSCs relieved AS by decreasing the number of infiltrating macrophages and the lipid level in plaques.

iPSC-MSCs affect the level of serum lipids and body weight. Because AS is also influenced by dyslipidemia, the authors measured serum lipid levels and body weight in each of the four groups (Fig. 3). Interestingly, although it was not identified that iPSC-MSCs had a significant effect on body weight (Fig $3 \mathrm{~B}, \mathrm{P}>0.05$ ), however the authors did find an effect on total cholesterol (TC), LDL and HDL levels, which were lower in the iPSC-MSC group than in the HFD group (Fig 3A; $\mathrm{P}<0.05$ ). There was no difference in these parameters between the $\mathrm{HF}$ and PBS groups (Fig 3A; P>0.05). In addition, the authors measured the lipid droplets in liver tissue (Fig 3C) and, consistent with the serum lipid result, the area of hepatic lipid droplets was significantly decreased by iPSC-MSCs (Fig. 3D; $\mathrm{P}<0.05$ ). Hence, the data demonstrated that the effects of iPSC-MSCs on AS may involve an ameliorating effect on lipid disorders.

iPSC-MSCs decreased the levels of inflammatory cytokines in ApoE KO mice. To investigate the effects of iPSC-MSCs on inflammatory responses in AS, the authors used ELISA kits to measure the expression levels of TNF- $\alpha$ and IL-6, two typical inflammatory cytokines, in an AS model (Fig. 4).
Consistent with the results of previous studies, the authors report that a HFD resulted in dramatically higher levels of TNF- $\alpha$ and IL- 6 in the ApoE KO mice than in the RD mice $(\mathrm{P}<0.01)$. However, iPSC-MSC transplantation resulted in significantly lower levels of HFD-induced TNF- $\alpha$ and IL-6 $(\mathrm{P}<0.05)$. Moreover, treating the mice with PBS alone had no effect on the levels of these cytokines when they were compared to the HFD groups $(\mathrm{P}>0.05)$. These results indicated that iPSC-MSCs decreased AS-related pathological inflammatory reactions by reducing the circulating levels of the pro-inflammatory cytokines TNF- $\alpha$ and IL-6.

iPSC-MSCs attenuate AS via Notch1. To further explore the effects of iPSC-MSCs on inflammatory responses in AS plaques, the authors measured the expression level of Notch1. In the present study, the expression of Notch1 was higher in the HFD and PBS groups than in the RD group $(\mathrm{P}<0.01)$. These data indicated that Notch1 was involved in AS. However, the iPSC-MSC group displayed markedly lower levels of Notch1 than were observed in the HFD group. Overall, infusions of iPSC-MSCs reduced the expression of Notch1 in AS plaques, which may have potentially contributed to the observed alleviation of AS inflammation (Fig. 5; $\mathrm{P}<0.05$ ).

\section{Discussion}

The anti-inflammatory effects and immunomodulatory functions of MSCs make them a promising therapeutic option for many diseases. Currently, the main source of MSCs is 


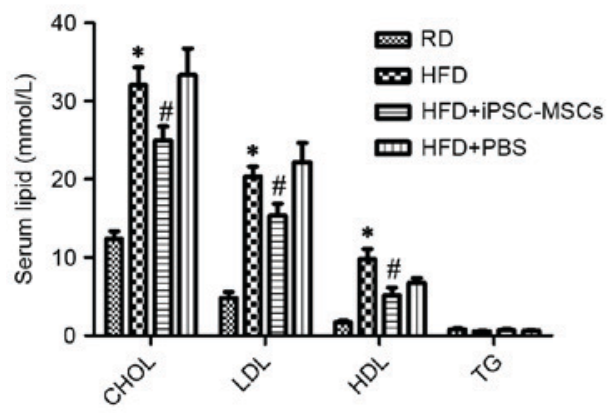

C

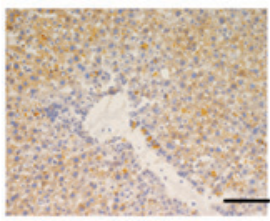

RD

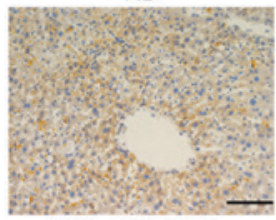

HFD+iPSC-MSCs

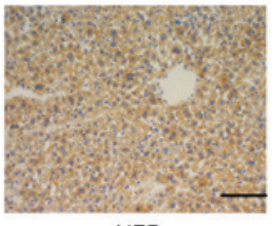

HFD

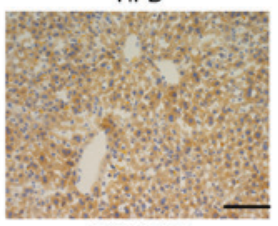

HFD+PBS
B

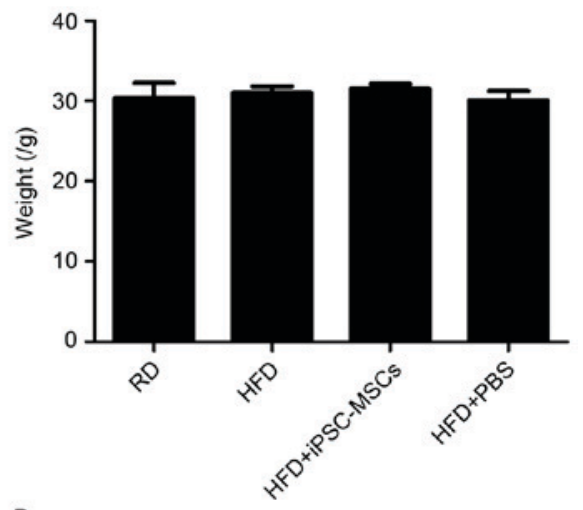

D

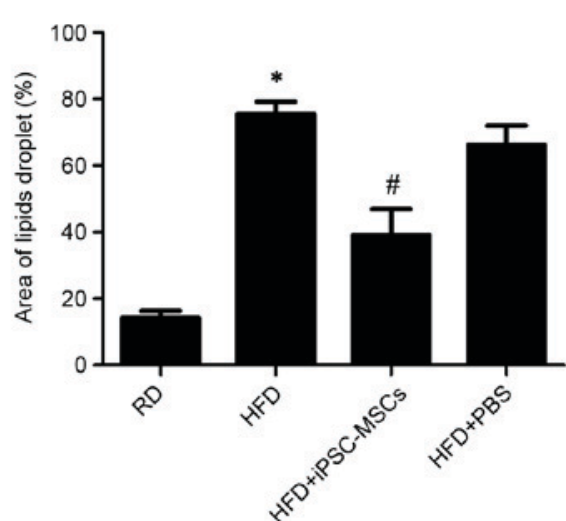

Figure 3. iPSC-MSCs' effect on the level of serum lipid, body weights and lipid droplets in liver tissue. (A) The levels of serum lipid of ApoE KO mice. (B) The weights of ApoE KO mice. (C) Representative photomicrographs of hepatic lipid droplets accumulation in ApoE KO mice. (D) Quantitative analysis of hepatic lipid droplets. Data are presented as mean \pm standard error of the mean. ${ }^{*} \mathrm{P}<0.01$ vs. RD group, ${ }^{\# P}<0.05$ vs. HFD group. Scale bar, $200 \mu \mathrm{m}$. iPSC-MSCs, induced pluripotent stem cell-derived mesenchymal stem cells; RD, regular diet group; HFD, high-fat diet group; PBS, phosphate-buffered saline; CHOL, cholesterol; LDL, low-density lipoprotein; HDL, high-density lipoprotein; TG, triglyceride.

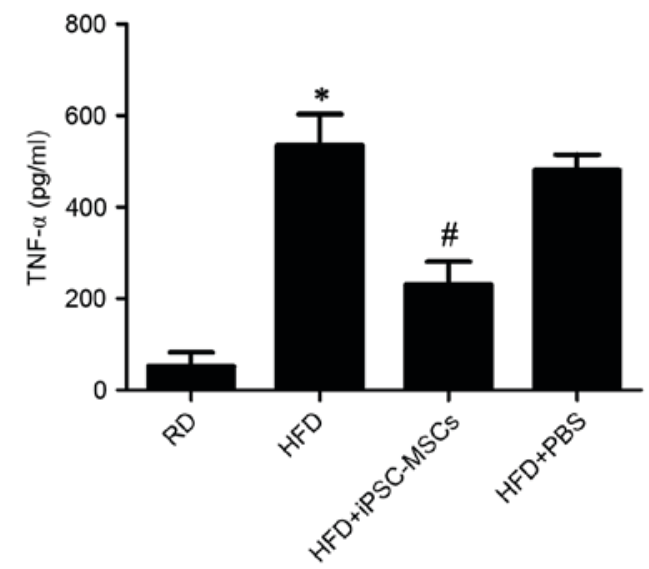

B

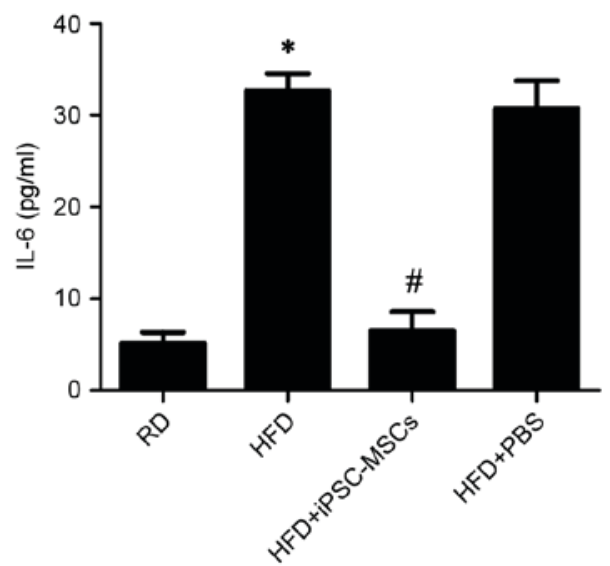

Figure 4. iPSC-MSCs decreased the level of inflammatory cytokines in ApoE KO mice. (A) TNF- $\alpha$ and (B) IL-6 levels in each group. Data are presented as mean \pm standard error of the mean. ${ }^{*} \mathrm{P}<0.01$ vs. RD group; ${ }^{~} \mathrm{P}<0.01$ vs. HFD group. iPSC-MSCs, induced pluripotent stem cell-derived mesenchymal stem cells; TNF- $\alpha$, tumor necrosis factor- $\alpha$; IL-6, interleukin; RD, regular diet group; HFD, high-fat diet group; PBS, phosphate-buffered saline.

the BM. However, there are several disadvantages associated with BM MSCs that restrict their clinical usefulness $(11,12)$. In addition, iPSC-MSCs could maintain stability during proliferation. Therefore, it is reasonable to assume that iPSC-MSCs may be a better candidate for AS stem cell therapies than BM-MSCs.
In the current study, the authors demonstrated that iPSC-MSCs significantly decreased the areas of AS plaques, inhibited infiltration by macrophages and lipid levels in plaques, decreased cholesterol levels in the serum, and reduced circulating levels of TNF- $\alpha$ and IL- 6 in ApoE KO mice. These data are consistent with previous studies that have 


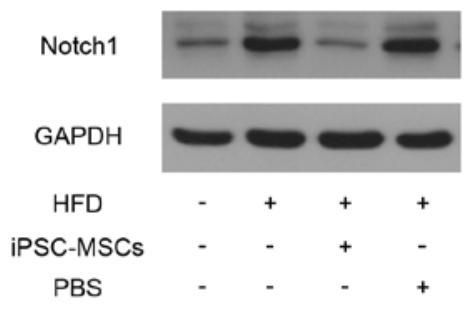

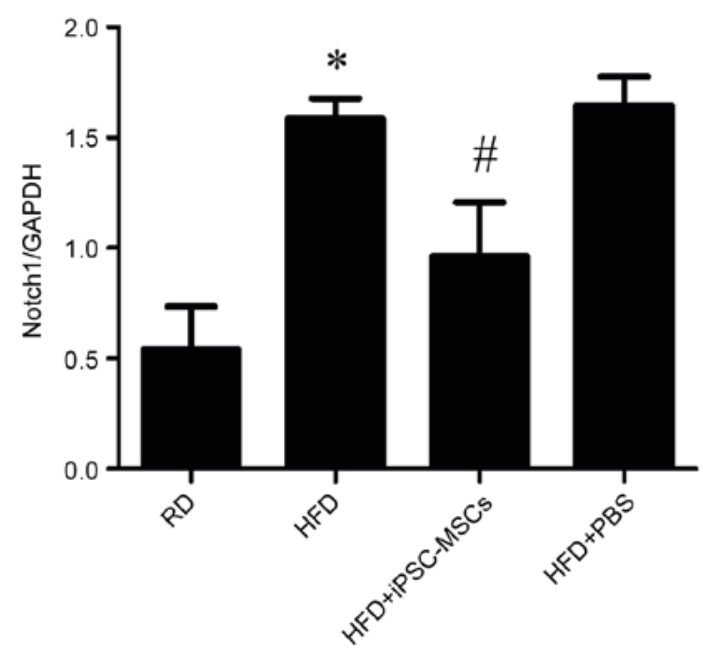

Figure 5. iPSC-MSCs attenuate atherosclerosis via Notch1. (A) Western blot analysis of Notch1 in each group. (B) Quantification of Notch1 protein level normalized to GAPDH. Data are presented as mean \pm standard error of the mean. $\mathrm{P}<0.01$ vs. RD group; ${ }^{\#} \mathrm{P}<0.05$ vs. HFD group. iPSC-MSCs, induced pluripotent stem cell-derived mesenchymal stem cells; RD, regular diet group; HFD, high-fat diet group.

indicated that MSCs derived from BM or other tissues also attenuated AS in AS mouse models (7,10,20). Furthermore, the mechanisms underlying the ability of iPSC-MSCs to alleviate AS were explored. The iPSC-MSC-mediated reduction in AS inflammation was accompanied by low protein levels of Notch1, indicating that iPSC-MSCs may reduce AS symptoms by affecting the Notch signaling pathway. Because iPSC-MSCs possess the ability to maintain stability during proliferation and do not lose this ability easily as they age, it is reasonable to assume that iPSC-MSCs may be a better candidate for AS stem cell therapies than other types of MSCs. To the best of the authors' knowledge, the present study is the first to demonstrate that iPSC-MSCs have been demonstrated to be capable of modulating AS in ApoE KO mice.

It is widely acknowledged that the development of AS is closely associated with macrophages and macrophage-derived foam cells. Once they have taken up lipids, macrophages transform into foam cells, which secrete many different inflammatory cytokines that can aggravate AS (21). Furthermore, a high accumulation of macrophages and lipids is characteristic of an advanced plaque, and these plaques are vulnerable to rupture (22). Moreno et al (23) indicated that atherosclerotic plaques with more macrophage-rich areas were more frequently observed in patients with unstable angina and non-Q-wave myocardial infarction. Hence, it is important to identify methods to reduce the number of macrophages in plaques to make the plaques more stable. To identify the effects of iPSC-MSCs on AS plaques, the authors measured the areas and analyzed the morphologies of plaques. Consistent with a previous report (24), the plaques in ApoE KO mice fed on a HFD were larger in size and contained higher levels of lipids and higher numbers of macrophages. In contrast, following iPSC-MSC transplantation, the plaques displayed improvements: They were smaller in size and were infiltrated by fewer lipids and macrophages. These data demonstrated that intravenously administering iPSC-MSCs not only reduced the sizes of plaques but also alleviated infiltration by inflammatory cells, which stabilized vulnerable plaques.
Blood cholesterol concentrations are closely associated with cardiovascular diseases, and decreasing blood cholesterol levels may therefore reduce the incidence of major coronary events (25). Additionally, hyperlipemia may aggravate the development of AS by increasing the circulating levels of proinflammatory mediators (26). Frodermann et al (7) demonstrated that BM-MSCs decreased serum levels of cholesterol in ApoE KO mice that were fed a HFD for 8 weeks by reducing the level of LPL in the liver. LPL hydrolyses triglycerides into free fatty acids, which are required for very low-density lipoprotein (VLDL). Similar to the results of previous studies, we also found that iPSC-MSCs lowered cholesterol levels in ApoE KO mice fed a HFD. Unfortunately, whether iPSC-MSCs alleviate hyperlipidemia by downregulating LPL levels in the liver remains uncertain. In addition, authors measured the level of HDL in the study. It was used to consider that there is an inverse association between amounts of HDL cholesterol and coronary heart disease (CHD) (27). However, many studies have reported that under some disease conditions HDL can become dysfunction and lose its atheroprotective property $(28,29)$. In AS or systemic inflammation, HDL can turn into pro-inflammatory states and promote monocyte chemotaxis $(30,31)$. Ansell et al (32) also identified that patients who developed CHD have very high levels of HDL, and exhibited high levels of pro-inflammatory HDL as well. Whether iPSC-MSCs allay AS inflammation through reducing pro-inflammatory HDL is still unclear.

TNF- $\alpha$ and IL- 6 are strong inflammatory cytokines that are closely associated with AS. TNF- $\alpha$ and IL- 6 both aggravate AS on their own and promote inflammatory cells to accumulate in AS plaques (20). In addition, the levels of TNF- $\alpha$, IL- 6 and other inflammatory cytokines are also significantly higher in many patients who are diagnosed with acute coronary syndrome and in animal models of this conditions $(20,33,34)$. In the current study, the authors determined that the expression levels of these cytokines were dramatically higher in ApoE KO mice fed on a HFD. Interestingly, transplanting iPSC-MSCs reduced serum levels of TNF- $\alpha$ and 
IL-6, which were otherwise induced by HFD. However, their levels remained higher than those observed in the RD group. Similarly, Li et al (20) demonstrated that LPS-stimulated macrophages produced less TNF- $\alpha$ when co-cultured with MSCs derived from the skin. Moreover, it was also reported that MSCs downregulated the expression of IL- 6 and TNF- $\alpha$ in ApoE KO mice (35). The authors therefore concluded that iPSC-MSCs alleviated AS inflammation by reducing the levels of specific inflammatory cytokines, such as IL- 6 and TNF- $\alpha$.

Notch1 levels was measured in plaques. Notch1 is a receptor in the Notch pathway. The Notch signaling pathway is a highly conserved pathway that is associated with many cellular processes, such as differentiation, proliferation and apoptosis (36). Recently, Liu et al (37) revealed that Notch1 was expressed at significantly higher levels in atherosclerotic lesions in the aortas of ApoE KO mice fed a HFD and in plaques in human patients. The Notch signaling inhibitor DAPT decreased the areas of plaques (38). Additionally, the Notch pathway is a modulator of inflammation (38). $\mathrm{Xu}$ et al (39) revealed that activating Notch1 promoted macrophages to polarize toward an inflammatory phenotype and to produce inflammatory cytokines. In the present study, the authors indicated that Notch1 expression was lower in the HFD + iPSC-MSCs group than in the HFD group, suggesting that iPSC-MSCs inhibited Notch1 expression. This finding could potentially lead to therapeutic strategies aimed at suppressing inflammatory reactions in ApoE KO mice.

There are some limitations to the current study. First, while the authors demonstrated that iPSC-MSCs alleviated AS, they failed to detect oblivious GFP-labeled iPSC-MSCs accumulated in plaques in ApoE KO mice. A different study failed to detect MSCs in plaques, and it has been demonstrated that MSCs exert their therapeutic potential mainly via paracrine effects, like secreting IL-8 (10). Zhang et al (18) have proven that iPSC-MSCs attenuated doxorubicin-induced cardiomyopathy through potent paracrine effects and listed TOP 10 secreted factors in iPSC-MSCs. In addition, a multitude of studies have demonstrated that $80-90 \%$ grafted cells die following cell transplantation due to oxidative stress or inflammation (40-42). Thus, it is reasonable to assume that direct effects make limited contribution to the therapeutic effects of iPSC-MSCs for AS and some other mechanisms may be involved, including the paracrine effects. However, the cytokines that are involved in this phenomenon will need to be identified in future studies. Secondly, iPSC-MSCs reduced the HFD-induced increase in the expression of Notch1. However, the authors did not explore the relationship between the Notch signaling pathway and inflammatory factors at the cellular level. In the future, the authors intend to analyze this relationship. In addition, immunological rejection following systematic administration of iPSC-MSCs is lacking in the present study. However, a study of human MSCs from adult tissues in animals without using immunosuppressants has also been reported (16). The authors acknowledged that immune rejection may occur in the current study, immunocompromized mice will be used in their future studies. Furthermore, AS is a complex process involving innate and adaptive immune mechanism (43). Whether the immune responses, like $\mathrm{T}$ cell responses or interferon- $\gamma$ produced by $\mathrm{T}$ helper 1 cells, are involved requires future study.
In conclusion, these findings clearly demonstrated that iPSC-MSCs suppress the formation of AS plaques. This effect involves a reduction in inflammatory responses and serum cholesterol concentrations in addition to a decrease in the expression of Notch1. iPSC-MSC transplantations may therefore be a promising treatment option in patients with a high AS burden.

\section{Acknowledgements}

The authors would like to thank Dr Qizhou Lian for generously providing us iPSC-MSCs. The present study was supported by the Natural Science Foundation of Guangdong Province (grant no. 2014A030313206).

\section{References}

1. Hansson GK and Libby P: The immune response in atherosclerosis: A double-edged sword. Nat Rev Immunol 6: 508-519, 2006.

2. Hansson GK: Inflammation, atherosclerosis, and coronary artery disease. N Engl J Med 352: 1685-1695, 2005.

3. Bobryshev YV: Monocyte recruitment and foam cell formation in atherosclerosis. Micron 37: 208-222, 2006.

4. Mangge H, Hubmann H, Pilz S, Schauenstein K, Renner W and März W: Beyond cholesterol-inflammatory cytokines, the key mediators in atherosclerosis. Clin Chem Lab Med 42: 467-474, 2004.

5. Kleemann R, Zadelaar S and Kooistra T: Cytokines and atherosclerosis: A comprehensive review of studies in mice. Cardiovasc Res 79: 360-376, 2008.

6. Singer NG and Caplan AI: Mesenchymal stem cells: Mechanisms of inflammation. Annu Rev Pathol 6: 457-478, 2011.

7. Frodermann V, van Duijn J, van Pel M, van Santbrink PJ, Bot I, Kuiper J and de Jager SC: Mesenchymal stem cells reduce murine atherosclerosis development. Sci Rep 5: 15559, 2015.

8. Bura A, Planat-Benard V, Bourin P, Silvestre JS, Gross F, Grolleau JL, Saint-Lebese B, Peyrafitte JA, Fleury S, Gadelorge M, et al: Phase I trial: The use of autologous cultured adipose-derived stroma/stem cells to treat patients with non-revascularizable critical limb ischemia. Cytotherapy 16: 245-257, 2014.

9. Vega A, Martin-Ferrero MA, Del Canto F, Alberca M, García V, Munar A, Orozco L, Soler R, Fuertes JJ, Huguet M, et al: Treatment of knee osteoarthritis with allogeneic bone marrow mesenchymal stem cells: A randomized controlled trial. Transplantation 99: 1681-1690, 2015.

10. Lin YL, Yet SF, Hsu YT, Wang GJ and Hung SC: Mesenchymal stem cells ameliorate atherosclerotic lesions via restoring endothelial function. Stem Cells Transl Med 4: 44-55, 2015.

11. Wagner W, Bork S, Horn P, Krunic D, Walenda T, Diehlmann A, Benes V, Blake J, Huber FX, Eckstein V, et al: Aging and replicative senescence have related effects on human stem and progenitor cells. PLoS One 4: e5846, 2009.

12. Kretlow JD, Jin YQ, Liu W, Zhang WJ, Hong TH, Zhou G, Baggett LS, Mikos AG and Cao Y: Donor age and cell passage affects differentiation potential of murine bone marrow-derived stem cells. BMC Cell Biol 9: 60, 2008.

13. Yu J, Vodyanik MA, Smuga-Otto K, Antosiewicz-Bourget J, Frane JL, Tian S, Nie J, Jonsdottir GA, Ruotti V, Stewart R, et al: Induced pluripotent stem cell lines derived from human somatic cells. Science 318: 1917-1920, 2007.

14. Takahashi K, Tanabe K, Ohnuki M,Narita M,Ichisaka T, Tomoda K and Yamanaka S: Induction of pluripotent stem cells from adult human fibroblasts by defined factors. Cell 131: 861-872, 2007.

15. Lian Q, Zhang Y, Zhang J, Zhang HK, Wu X, Zhang Y, Lam FF, Kang S, Xia JC, Lai WH, et al: Functional mesenchymal stem cells derived from human induced pluripotent stem cells attenuate limb ischemia in mice. Circulation 121: $1113-1123,2010$

16. Sun YQ, Deng MX, He J, Zeng QX, Wen W, Wong DS, Tse HF, $\mathrm{Xu} \mathrm{G}$, Lian Q, Shi J and Fu QL: Human pluripotent stem cell-derived mesenchymal stem cells prevent allergic airway inflammation in mice. Stem Cells 30: 2692-2699, 2012.

17. Diederichs S and Tuan RS: Functional comparison of human-induced pluripotent stem cell-derived mesenchymal cells and bone marrow-derived mesenchymal stromal cells from the same donor. Stem Cells Dev 23: 1594-1610, 2014. 
18. Zhang Y, Liang X, Liao S, Wang W, Wang J, Li X, Ding Y, Liang Y, Gao F, Yang M, et al: Potent paracrine effects of human induced pluripotent stem cell-derived mesenchymal stem cells attenuate doxorubicin-induced cardiomyopathy. Sci Rep 5: $11235,2015$.

19. Zhang QZ, Su WR, Shi SH, Wilder-Smith P, Xiang AP, Wong A, Nguyen AL, Kwon CW and Le AD: Human gingiva-derived mesenchymal stem cells elicit polarization of $\mathrm{m} 2$ macrophages and enhance cutaneous wound healing. Stem Cells 28: 1856-1868, 2010.

20. Li Q, Sun W, Wang X, Zhang K, Xi W and Gao P: Skin-derived mesenchymal stem cells alleviate atherosclerosis via modulating macrophage function. Stem Cells Transl Med 4: 1294-1301, 2015.

21. Galkina $\mathrm{E}$ and Ley $\mathrm{K}$ : Immune and inflammatory mechanisms of atherosclerosis (*). Annu Rev Immunol 27: 165-197, 2009.

22. Stary HC, Chandler AB, Dinsmore RE, Fuster V, Glagov S, Insull W Jr, Rosenfeld ME, Schwartz CJ, Wagner WD and Wissler RW: A definition of advanced types of atherosclerotic lesions and a histological classification of atherosclerosis. A report from the committee on vascular lesions of the council on arteriosclerosis, American heart association. Arterioscler Thromb Vasc Biol 15: 1512-1531, 1995.

23. Moreno PR, Falk E, Palacios IF, Newell JB, Fuster V and Fallon JT: Macrophage infiltration in acute coronary syndromes. Implications for plaque rupture. Circulation 90: 775-778, 1994.

24. Zhou J, Lhoták S, Hilditch BA and Austin RC: Activation of the unfolded protein response occurs at all stages of atherosclerotic lesion development in apolipoprotein E-deficient mice. Circulation 111: 1814-1821, 2005.

25. Baigent C, Keech A, Kearney PM, Blackwell L, Buck G, Pollicino C, Kirby A, Sourjina T, Peto R, Collins R and Simes R Cholesterol Treatment Trialists' (CTT) Collaborators: Efficacy and safety of cholesterol-lowering treatment: Prospective meta-analysis of data from 90,056 participants in 14 randomised trials of statins. Lancet 366: 1267-1278, 2005.

26. van Diepen JA, Berbée JF, Havekes LM and Rensen PC: Interactions between inflammation and lipid metabolism: Relevance for efficacy of anti-inflammatory drugs in the treatment of atherosclerosis. Atherosclerosis 228: 306-315, 2013.

27. Besler C, Heinrich K, Rohrer L, Doerries C, Riwanto M, Shih DM, Chroni A, Yonekawa K, Stein S, Schaefer N, et al: Mechanisms underlying adverse effects of HDL on eNOS-activating pathways in patients with coronary artery disease. J Clin Invest 121: 2693-2708, 2011

28. Vuilleumier N, Dayer JM, von Eckardstein A and Roux-Lombard P: Pro- or anti-inflammatory role of apolipoprotein A-1 in high-density lipoproteins? Swiss Med Wkly 143: w13781, 2013.

29. Rizzo M, Otvos J, Nikolic D, Montalto G, Toth PP and Banach M Subfractions and subpopulations of HDL: An update. Curr Med Chem 21: 2881-2891, 2014.

30. Von Feldt JM: High-density lipoprotein: Does it have a dark side? Arthritis Res Ther 10: 121, 2008.
31. Skaggs BJ, Hahn BH, Sahakian L, Grossman J and McMahon M: Dysfunctional, pro-inflammatory HDL directly upregulates monocyte PDGFR $\beta$, chemotaxis and $\mathrm{TNF} \alpha$ production. Clin Immunol 137: 147-156, 2010.

32. Ansell BJ, Fonarow GC and Fogelman AM: The paradox of dysfunctional high-density lipoprotein. Curr Opin Lipidol 18: 427-434, 2007.

33. Khan DA, Ansari WM and Khan FA: Pro/anti-inflammatory cytokines in the pathogenesis of premature coronary artery disease. J Interferon Cytokine Res 31: 561-567, 2011.

34. Wang XH, Liu SQ, Wang YL and Jin Y: Correlation of serum high-sensitivity C-reactive protein and interleukin-6 in patients with acute coronary syndrome. Genet Mol Res 13: 4260-4266, 2014.

35. Hashizume R, Yamawaki-Ogata A, Ueda Y, Wagner WR and Narita Y: Mesenchymal stem cells attenuate angiotensin II-induced aortic aneurysm growth in apolipoprotein E-deficient mice. J Vasc Surg 54: 1743-1752, 2011.

36. Artavanis-Tsakonas S, Rand MD and Lake RJ: Notch signaling: Cell fate control and signal integration in development. Science 284: 770-776, 1999.

37. Liu ZJ, Tan Y, Beecham GW, Seo DM, Tian R, Li Y, Vazquez-Padron RI, Pericak-Vance M, Vance JM, Goldschmidt-Clermont PJ, et al: Notch activation induces endothelial cell senescence and pro-inflammatory response: Implication of Notch signaling in atherosclerosis. Atherosclerosis 225: 296-303, 2012.

38. Aquila G, Pannella M, Morelli MB, Caliceti C, Fortini C, Rizzo P and Ferrari R: The role of Notch pathway in cardiovascular diseases. Glob Cardiol Sci Pract 2013: 364-371, 2013.

39. Xu H, Zhu J, Smith S, Foldi J, Zhao B, Chung AY, Outtz H, Kitajewski J, Shi C, Weber S, et al: Notch-RBP-J signaling regulates the transcription factor IRF8 to promote inflammatory macrophage polarization. Nat Immunol 13: 642-650, 2012.

40. Qiu X, Zhang S, Zhao X, Fu K and Guo H: The therapeutic effect of adipose-derived mesenchymal stem cells for radiation-induced bladder injury. Stem Cells Int 2016: 3679047, 2016.

41. Müller-Ehmsen J, Whittaker P, Kloner RA, Dow JS, Sakoda T, Long TI, Laird PW and Kedes L: Survival and development of neonatal rat cardiomyocytes transplanted into adult myocardium. J Mol Cell Cardiol 34: 107-116, 2002.

42. Toma C, Pittenger MF, Cahill KS, Byrne BJ and Kessler PD: Human mesenchymal stem cells differentiate to a cardiomyocyte phenotype in the adult murine heart. Circulation 105: 93-98, 2002.

43. Pushpan CK, V S, G S, Rathnam P, A J and A H: Attenuation of atherosclerotic complications by modulating inflammatory responses in hypercholesterolemic rats with dietary Njavara rice bran oil. Biomed Pharmacother 83: 1387-1397, 2016.

This work is licensed under a Creative Commons

Attribution-NonCommercial-NoDerivatives 4.0

International (CC BY-NC-ND 4.0) License. 